\title{
Glutathione Disulfide NOV-002
}

\author{
National Cancer Institute
}

\section{Source}

National Cancer Institute. Glutathione Disulfide NOV-002. NCI Thesaurus. Code C62537.

A stabilized formulation of disodium glutathione disulfide (GSSG; oxidized glutathione) and cisplatin (1000:1) with potential chemoprotective and immunomodulating activities. Mimicking endogenous GSSG, glutathione disulfide NOV-002 acts as a competitive substrate for gamma-glutamyl-transpeptidase (GGT), which may result in the Sglutathionylation of proteins, predominantly actin, a redox stress on endoplasmic reticulum (ER), and ER stress-induced apoptosis; S-glutathionylation may be stimulated by reactive oxyg en species (ROS) liberated by a glutathione disulfide NOV-002-induced increase in GGT activity. Glutathione disulfide NOV-002 may also induce phosphorylation of proteins such as ERK and p38, two kinases that play critical regulatory roles in cell proliferation and apoptosis. The cisplatin component of this agent does not provide an effective therapeutic concentration of cisplatin in vivo. 